\title{
Compression and shearing force on kernel rupture in shelling fresh lotus seeds
}

\author{
Jincheng He*, Zhiying Tao, Shihua Liang, Dapeng Ye \\ (College of Mechanical and Electrical Engineering, Fujian Agriculture and Forestry University, Fuzhou 350002, China)
}

\begin{abstract}
The mechanical properties of fresh lotus seeds are still poorly understood, which complicates the design of mechanical shelling machinery. Therefore, this work carried out four-factor orthogonal tests to determine the maximum permitted compressive force and minimum necessary shearing force to shell fresh lotus seeds without rupturing the kernel. It was found that the mean compression force that cracked the fresh lotus seed and led to kernel rupture was $213.03 \mathrm{~N}$. Both the compressive force and the seed deformation upon kernel rupture were affected, in descending order of significance, by loading mode, seed grade, loading rate, and seed standing time. On the other hand, the shearing force needed to shell the seeds had a mean value of $7.84 \mathrm{~N}$, far less than the compressive force that cracked the seed shell. The shearing force was affected, in descending order of significance, by seed standing time, tip angle of cutter blade, and loading rate, but not significantly affected by seed grade. The results suggested that mechanical shelling of fresh lotus seeds should ideally be carried out for fresh lotus seeds with a standing time of no more than $6 \mathrm{~h}$ using a cutter blade with an angle of about $40^{\circ}$ at a loading rate of $30-90 \mathrm{~mm} / \mathrm{min}$.

Keywords: shelling fresh lotus seeds, mechanical properties, shearing, compression, kernel rupture
\end{abstract}

DOI: $10.25165 /$ j.ijabe.20211401.5193

Citation: He J C, Tao Z Y, Liang S H, Ye D P. Compression and shearing force on kernel rupture in shelling fresh lotus seeds. Int J Agric \& Biol Eng, 2021; 14(1): 237-242.

\section{Introduction}

Lotus seed is highly nutritious and contains abundant fat, $\operatorname{starch}^{[1]}$, flavonoids ${ }^{[2,3]}$, and other functional substances. The annual production of lotus seed in the Fujian Province reached $12205 \mathrm{t}$ in $2017^{[4]}$, corresponding to a GDP of about 1.8 billion Chinese Yuan. The highly popular "Jianlian" variety of lotus seeds is mainly produced in Jianning, Wuyishan, and other localities in Fujian. Jianlian is also known as the "Decored White Lotus", since it is processed from fresh lotus via multiple steps including shelling, membrane removal, coring, drying, etc. ${ }^{[5]}$ Shelling is a highly important step in processing fresh lotus seeds and directly affects the appearance and the price of the finished product, because the delicate seed is susceptible to damages from the squeeze, scratch, etc. The damaged seed will turn yellow and wrinkle after drying and become less tasty, and will without any doubt sell poorly. As of today, mechanical shelling damages lotus seeds much more easily than manual shelling, and this situation has seriously obstructed the application of mechanized processing of fresh lotus seeds at a larger scale ${ }^{[6,7]}$.

Needless to say, the mechanical properties of materials are the starting point in developing and improving production machinery. Extensive studies have been dedicated to the mechanical properties of agricultural materials including lychee ${ }^{[8-10]}$, stalk ${ }^{[11-13]}$, peach $^{[14,15]}$, pomegranate ${ }^{[16]}$, hazelnut ${ }^{[17]}$, etc., and it was found that the mechanical properties of materials being processed depended

Received date: 2019-06-06 Accepted date: 2020-07-08

Biographies: Zhiying Tao, Master candidate, research interest: agricultural mechanization engineering, Email: 1440743305@qq.com; Shihua Liang, Master, research interest: agricultural mechanization engineering, Email: 302584954@qq.com; Dapeng Ye, PhD, Professor, research interest: control engineering, Email: fau_ydp@163.com.

*Corresponding: Jincheng He, PhD, Associate Professor, research interest: agricultural mechanization engineering. College of mechanical and electrical engineering, Fujian agriculture and forestry university, Fuzhou 350002, China. Tel: +86-18850117251, Email: jhe@fafu.edu.cn. greatly on externally applied forces. In the mechanical shelling process, the seed is pressed and rubbed to separate the kernel from the shell on which a circle had been cut by the blade. Regrettably, existing reports on the mechanical properties of lotus seeds during processing most focus on dry lotus seeds ${ }^{[18-21]}$, which differ significantly from those of fresh lotus seeds. Xie et al. ${ }^{[21]}$ established a finite element model to analyze lotus seeds under static positive pressure and assessed the stress and strain of lotus seeds under different loading modes. Ye et al. ${ }^{[19]}$ studied white lotus and red lotus with a dry shell to inspect their physical parameters such as density, water content, outer dimensions, etc. Zhao et al. ${ }^{[22]}$ compared the shell of dried red lotus seed with the chestnut shell to examine their microstructures and nano-mechanical properties.

With regards to the mechanical properties of fresh lotus seeds, Zhou et al. ${ }^{[23]}$ studied the physical and mechanical properties and cutting characteristics of lotus seeds, and Ma et al. ${ }^{[24,25]}$ studied the mechanical coring of fresh lotus and the mechanical properties of lotus kernel. However, neither investigated the material characteristics of the shell of fresh lotus seeds. Zhu et al. ${ }^{[26]}$ measured the shearing force needed to shell fresh lotus seeds as well as the firmness of the lotus kernel. Nevertheless, it remains to be clarified how these mechanical properties of fresh lotus seeds depend on variables such as standing time, loading mode, etc. Therefore, this work aims to study the compression and shearing properties of fresh lotus seeds in relation to loading mode, seed grade, loading rate, standing time, etc. to thus establish a theoretical basis for developing devices for mechanical shelling.

\section{Materials and methods}

\subsection{Materials}

The test samples (variety "Jianxuan \#17") were fresh lotus seeds produced at the Wufu Town of the Wuyishan City in the Fujian Province of China. The samples had an oval shape with the long axis and the short axis ranging in $23.20-26.20 \mathrm{~mm}$ and 
17.00-19.30 mm, respectively. They were tested on an AG-Xplus electronic universal testing machine (Shimadzu, Japan).

\subsection{Methods}

\subsubsection{Compression}

Lotus seeds are constantly under external compressive force during the shelling. Hence, the compression experiment aimed to determine the maximum force that the lotus kernel could withstand before it was damaged, so as to guide the design of gripping, pressing, and shelling devices in the future. Shell compression and kernel rupture were tested separately.

Shell rupture was tested by compressing fresh whole lotus seed under horizontal or vertical loading (Figure 1) until shell rupture to record the compressive force and the shell deformation during the process.

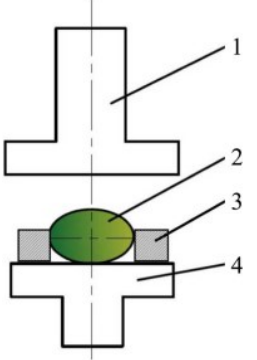

a.

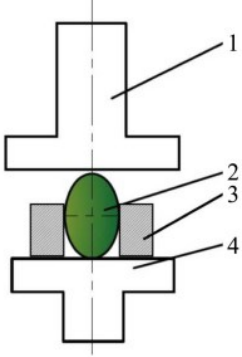

b.
1. Top plate 2. Lotus seed 3. Clamp 4. Bottom plate

Figure 1 Schematic diagram of (a) horizontal and (b) vertical compression of lotus seed

Kernel rupture was tested with fresh whole lotus seeds to examine the seed deformation as well as the compressive force that ruptured the kernel. The influences of the four factors were evaluated. The factors included the loading mode (factor A), the seed grade (factor B), the loading rate (factor $\mathrm{C}$ ), and the standing time of the lotus seed (factor D). With regards to loading mode, compression was applied either vertically (on the long axis) or horizontally (on the short axis). The grade of the lotus seed fell into four categories based on the length of its short axis, i.e., grade $\mathrm{S} 1$ for $<17.5 \mathrm{~mm}$, grade S2 for $17.5-18.1 \mathrm{~mm}$, grade $\mathrm{S} 3$ for 18.1-18.7 mm, and grade S4 for $>18.7 \mathrm{~mm}$. Four levels of loading rate were considered. The standing time of the seed, which also had had four levels, was the time elapsed since the seed had been peeled from fresh lotus and stored (at $28^{\circ} \mathrm{C}$ and $75 \%$ relative humidity). Fresh lotus was harvested on the same day of the experiment, and seeds that stood for less than two hours after peeling from fresh lotus were considered to have a standing time of $0 \mathrm{~h}$. Table 1 shows the detailed setting of the examined factors.

Table 1 Factors and levels of the orthogonal compression test of fresh lotus seeds

\begin{tabular}{ccccc}
\hline \multirow{3}{*}{ Level } & \multicolumn{4}{c}{ Factor } \\
\cline { 2 - 5 } & $\mathrm{A}$ & $\mathrm{B} / \mathrm{mm}$ & $\mathrm{C} / \mathrm{mm} \cdot \mathrm{min}^{-1}$ & $\mathrm{D} / \mathrm{h}$ \\
\hline 1 & Horizontal & $\mathrm{S} 1(<17.5)$ & 20 & 0 \\
2 & Vertical & $\mathrm{S} 2(17.5-18.1)$ & 40 & 17 \\
3 & & $\mathrm{~S} 3(18.1-18.7)$ & 60 & 24 \\
4 & & $\mathrm{~S} 4(>18.7)$ & 80 & 36 \\
\hline
\end{tabular}

A dummy-level orthogonal test design was adopted using the four-level $\mathrm{L}_{16}\left(4^{5}\right)$ orthogonal test scheme. Compression was carried out by placing the lotus seed on a static bottom plate and pressing it with a top plate from above (Figure 1). Each test was repeated with eight lotus seeds and the force-deformation curve during compression was recorded to determine the compressive rupture force. The influence of the four factors (i.e., loading mode, seed grade, loading rate, and standing time) was then evaluated.

\subsubsection{Shearing}

Shearing was examined by considering the following factors: the tip angle of the cutter blade (denoted as factor E), seed grade (factor B), loading rate (factor C), and standing time (factor D). Four blade angles were considered (Figure 2). Four grades of the lotus seed were considered similarly as described in the previous section. The loading rate and the standing time each considered eight levels as described in Table 2. An orthogonal test design using the $\mathrm{L}_{16}\left(2^{15}\right)$ scheme with the dummy-factor method was adopted (Table 3). Figure 3 shows the loading mode during the test. The lotus seed was fixed on the bottom plate, and the cutter blade moved downward to push into the shell and cut it open. Each test was repeated eight times (i.e., eight lotus seeds were measured) and both the shearing force and the shear stroke curve were recorded. The shearing force during shelling was determined accordingly, and the influence of the four factors was examined.

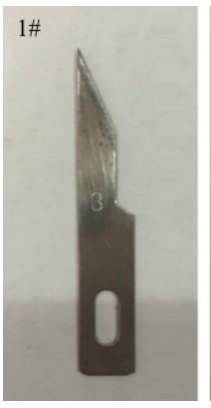

a. $25^{\circ}$

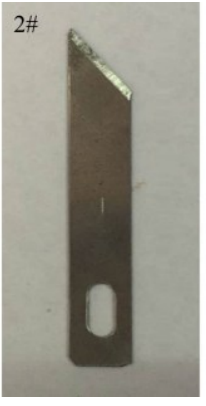

b. $40^{\circ}$

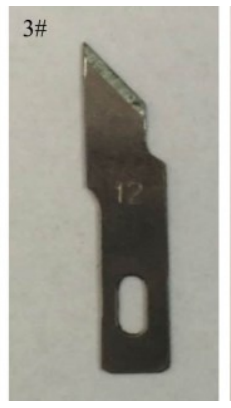

c. $45^{\circ}$

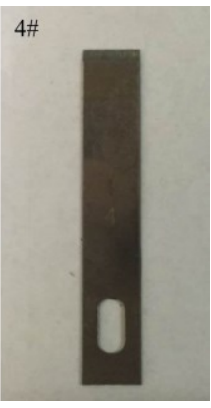

d. $90^{\circ}$
Figure 2 Cutter blade of various angles

Table 2 Factors and levels of the orthogonal test for the cutting test of fresh lotus seeds

\begin{tabular}{ccccc}
\hline & \multicolumn{3}{c}{ Factor } \\
\cline { 2 - 5 } & $\mathrm{E}$ & $\mathrm{B} / \mathrm{mm}$ & $\mathrm{C} / \mathrm{mm} \cdot \mathrm{min}^{-1}$ & $\mathrm{D} / \mathrm{h}$ \\
\hline 1 & $25^{\circ}$ & $\mathrm{S} 1(<17.5)$ & 10 & 0 \\
2 & $40^{\circ}$ & $\mathrm{S} 2(17.5-18.1)$ & 30 & 6 \\
3 & $45^{\circ}$ & $\mathrm{S} 3(18.1-18.7)$ & 50 & 9 \\
4 & $90^{\circ}$ & $\mathrm{S} 4(>18.7)$ & 70 & 12 \\
5 & & & 90 & 18 \\
6 & & & 110 & 24 \\
7 & & & 130 & 36 \\
8 & & & 150 & 48 \\
\hline
\end{tabular}

Table 3 Design of the $L_{16}\left(2^{15}\right)$ orthogonal cutting test

\begin{tabular}{ccccccc}
\hline Factor & Empty Column & B & D & E & C & Blank column \\
\hline $\begin{array}{l}\text { Column } \\
\text { Number }\end{array}$ & 4 & 2,7 & $3,14,15$ & 8,9 & $6,10,11$ & $1,5,12,13$ \\
\hline
\end{tabular}

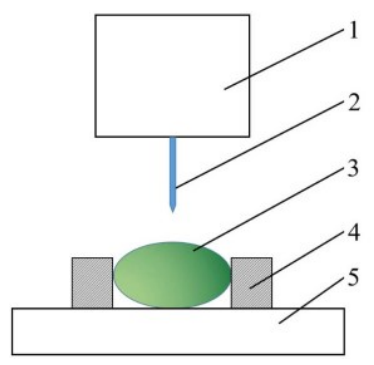

a.

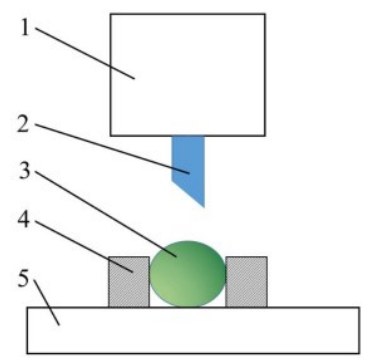

b.
1. Blade driver 2. Cutter blade 3. Lotus seed 4. Clamp 5. Bottom plate Figure 3 Schematic in (a) front view and (b) side view of lotus seed cutting 


\section{Results and discussion}

\subsection{Analysis of compression process}

\subsubsection{Kernel rupture and shell cracking under compression}

Appropriate shelling not only depends on the mechanical properties of the kernel and the shell but also the internal structure of the fresh lotus seed. Figure 4 shows that the fresh lotus seed is mainly composed of four parts, i.e., the shell, the membrane, the kernel, and the core. The membrane wraps tightly on the kernel. The shell is about 0.92-1.10 mm thick, and a gap of about 0.01-0.36 mm exists between the membrane and the shell. This gap makes it possible to shell the seed without damaging the kernel.

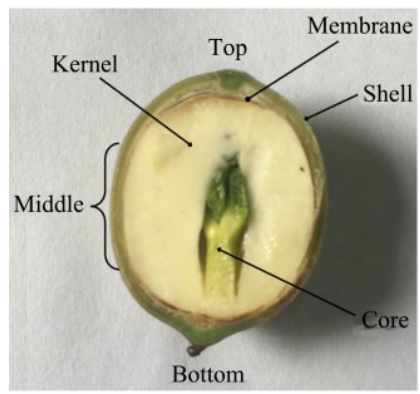

Figure 4 Sectional view of fresh lotus seed

Table 4 shows that the capacity of the seed to withstand compression was anisotropic and depended on the loading direction since the relative seed deformation and the instantaneous compressive force upon shell cracking were greater when loading was applied vertically than horizontally. Hence, fresh lotus seeds appeared to be more resistant to compression in the vertical direction than in the horizontal direction. Figure 5a shows that when the seed was compressed vertically, the cracks were parallel to the direction of loading and the initial cracks appeared in the middle of the seed. In contrast, Figures $5 b-5 d$ shows that when the seed was compressed horizontally, the cracking pattern was much more irregular in both position and direction. Specifically, the direction of the cracks appeared to be random, and the initial cracks appeared mostly in the middle but were also formed at the ends. The results showed that fresh lotus seed differed significantly from lychee, longan, etc. in terms of compression since the cracks on the shell of lychee and longan only extend along the direction of the compressive force and only appear in the middle ${ }^{[8,9,27]}$.

Table 4 Mechanical parameters of fresh lotus seed under compression

\begin{tabular}{ccc}
\hline Loading & $\begin{array}{c}\text { Instantaneous compressive } \\
\text { force/N* }\end{array}$ & $\begin{array}{c}\text { Relative seed deformation } \\
\text { /\%* }\end{array}$ \\
\hline Vertical & 275.10 & $32.06 \%$ \\
Horizontal & 150.95 & $25.17 \%$ \\
\hline
\end{tabular}

Note: * Values recorded at the instant of shell cracking

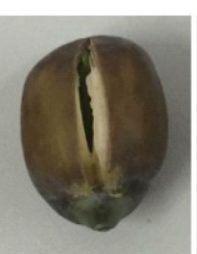

a.

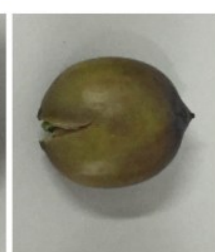

b.

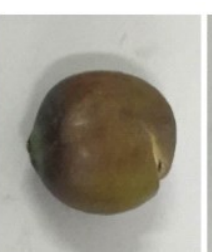

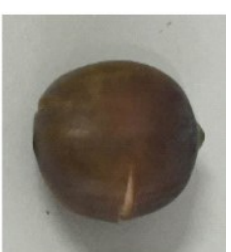

d.
Figure 5 Crack patterns of fresh lotus seeds subject to (a) vertical compression and $(b-d)$ horizontal compression

3.1.2 Seed deformation under compression

The fresh lotus seed is usually compressed horizontally in mechanical shelling. Figure 6 shows the relationship between seed deformation and compressive force during horizontal compression, where two obvious turning points could be noted. The first point corresponded to kernel rupture, which was associated with a sudden decrease of pressure. The pressure continued to increase with rising seed deformation afterward and it ultimately cracked the shell, i.e., the second point on the curve. The observed pattern differed from the situation of other fruits like lychee. It was reported that lychee showed only one turning point on its graph ${ }^{[9]}$, i.e., the lychee shell cracked and the pressure declined drastically as soon as the compressive force was enough for the shell to crack. This can be rationalized as follows. The lychee pulp is soft and has a higher water content $(80 \% \mathrm{wb}$ on average), whereas the kernel of fresh lotus seed is harder and has less water content $(61 \% \mathrm{wb}$ on average). Consequently, the lotus seed showed stronger resistance against pressure after kernel rupture with extra protection from the shell, which was why the sudden pressure drop upon kernel rupture was much less prominent. Figure 6 also showed that there was no obvious point of stress yield during compression. Regression between seed deformation (from zero to kernel rupture) and compressive force showed a significant linear correlation $\left(R^{2}=0.99197\right)$.

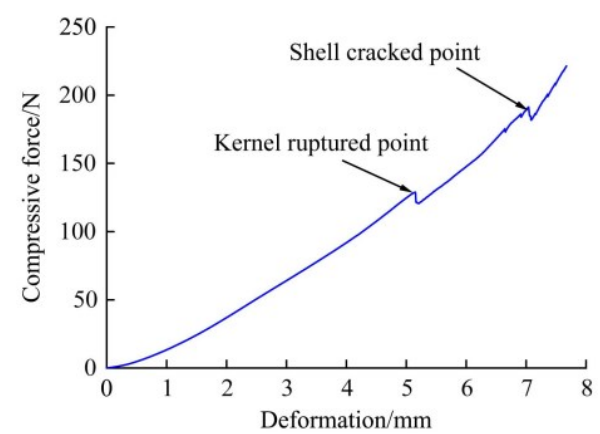

Figure 6 Relationship between seed deformation and compressive force during horizontal compression, where two obvious turning points could be noted (horizontal compression of grade S1 fresh

lotus seed at $20 \mathrm{~mm} / \mathrm{min}$, the standing time of which was $0 \mathrm{~h}$ )

\subsection{Orthogonal test of compression}

Figure $7 \mathrm{a}$ shows the instantaneous compressive force upon kernel rupture and Figure $7 \mathrm{~b}$ shows the corresponding seed deformation. The rupture force ranged in 102.90-337.10 $\mathrm{N}$ with a mean of $213.03 \mathrm{~N}$, and the seed deformation ranged in 3.51$9.41 \mathrm{~mm}$ with a mean of $6.26 \mathrm{~mm}$.

Variance analysis showed that the compressive rupture force was significantly influenced by the loading mode, seed grade, loading rate, and standing time ( $p<0.01$ for all four factors), with the degree of influence falling in the order of loading mode, loading rate, standing time, seed grade. Seed deformation was significantly influenced by loading mode $(p<0.01)$, loading rate $(p<0.01)$, standing time $(p<0.01)$, and seed grade $(p<0.05)$, and the degree of influence fell in the order of loading mode, standing time, loading rate, seed grade.

Figure 8 shows the impact of each factor on the compressive force and seed deformation upon kernel rupture. Figure 8a shows that for fresh lotus seeds, kernel rupture occurred more easily when compression was applied horizontally instead of, vertically when the lotus seed was of grade S1 instead of grade S4 (little difference was observed between grade S2 and grade S3), when the loading rate was high, and when the standing time was longer than $17 \mathrm{~h}$. A similar trend was observed for seed deformation in Figure $8 \mathrm{~b}$ as well. Since the lotus seed is porous, its shell starts to lose 
moisture when it is not protected by the lotus pod but exposed to air. As a result, the shell loses moisture sooner than the kernel and firstly softens, then toughens, and finally loses elasticity.

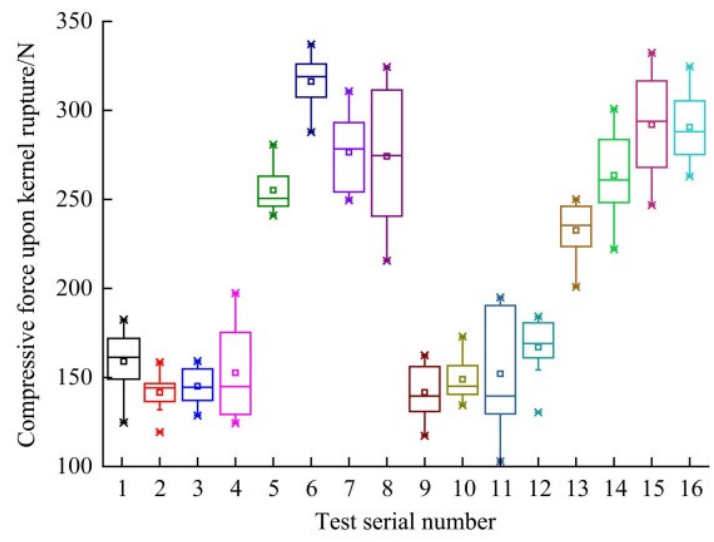

a. Compressive force upon kernel rupture
Nevertheless, after a standing time of $17 \mathrm{~h}$, the kernel also starts to lose moisture from its surface and becomes less brittle, which would then increases the compressive force upon kernel rupture.

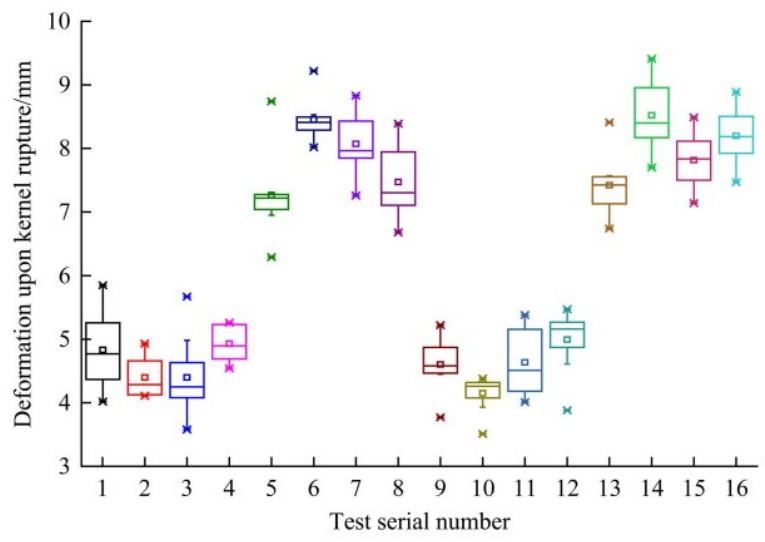

b. Seed deformation upon kernel rupture

Figure 7 Orthogonal test results of the compression of fresh lotus seeds influenced by the loading mode, seed grade, loading rate, and standing time
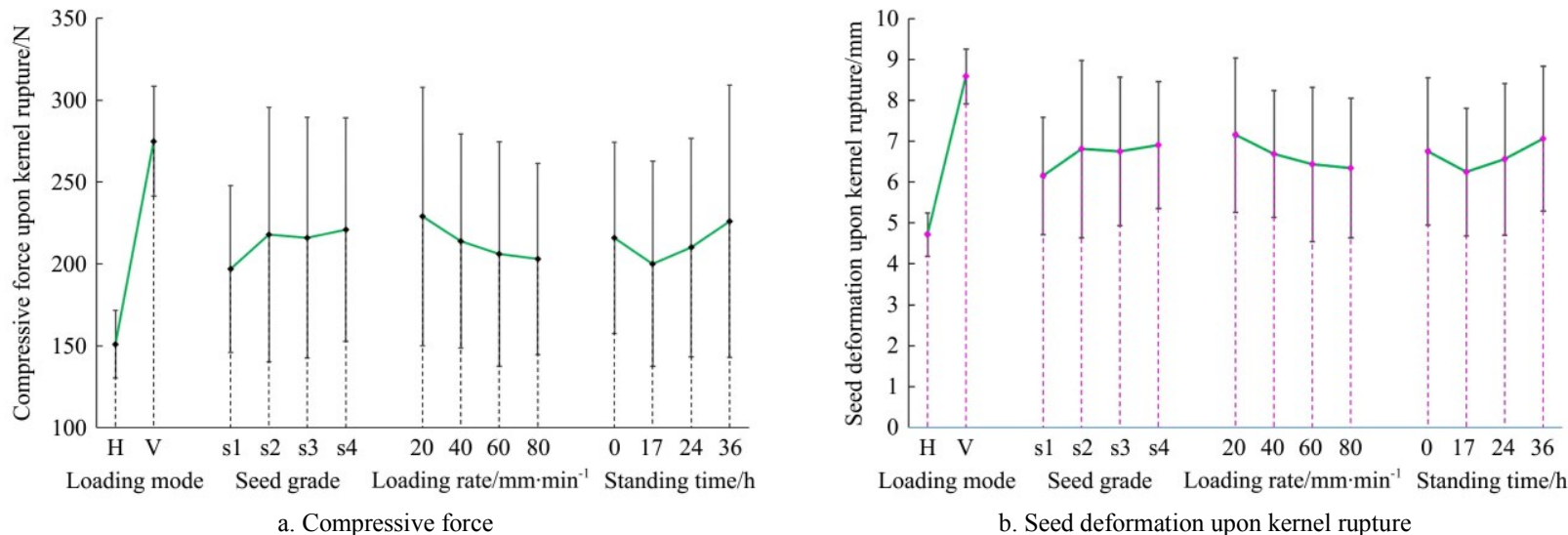

Figure 8 Impact of the factors of the loading mode, seed grade, loading rate, and standing time

\subsection{Orthogonal test of shearing}

Figure 9 showed that as the shear stroke increased, the shearing force increased until the blade cut through the shell, at which time the shearing force declined suddenly because of the gap between the shell and the kernel. When the stroke increased further, the blade cut into the kernel and the shearing force continued to increase. Hence, the shearing force at the turning point on the curve was considered as the shearing force of shelling.

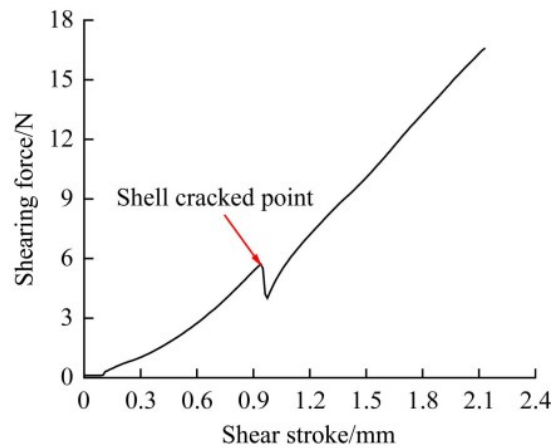

Figure 9 Relationship between seed shearing force and shear stroke

Figure 10 shows that the shearing force of shelling the seed ranged from 1.64-27.31 N with a mean of $7.84 \mathrm{~N}$, which was much smaller than the compressive force that cracked the seed. The shearing force was significantly affected by standing time, blade angle, and loading rate $(p<0.01$ for all), but not by seed grade $(p>0.05)$. The degree of influence is blade angle (factor E), standing time (factor D), loading rate (factor $\mathrm{C}$ ), and seed grade (factor B) in descending order.

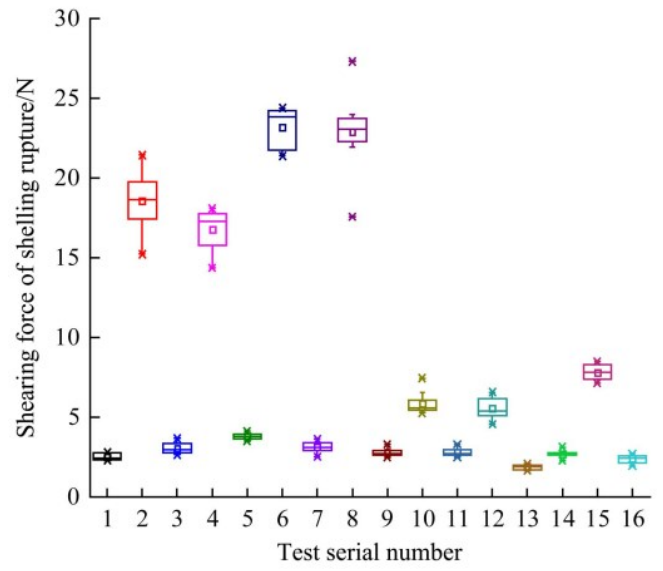

Figure 10 Orthogonal test results of shearing force of shelling rupture the fresh lotus seeds

It can be seen from Figure 11 that seed grade had a negligible influence on the shearing force. Among cutter blades of different angles, the mean shearing force of blade \#2 $\left(40^{\circ}\right)$ was the lowest, followed by blade \#1 $\left(25^{\circ}\right)$, blade \#3 $\left(45^{\circ}\right)$, and blade \#4 $\left(90^{\circ}\right)$.

Fisher's least significant difference (LSD) test of the shearing force showed that a significant difference existed between cutter blade \#4 and cutter blades \#1, \#2, and \#3, but not among cutter 
blades \#1, \#2, and \#3. Thus, a blade angle of approximately

$25^{\circ}-40^{\circ}$ was recommended.

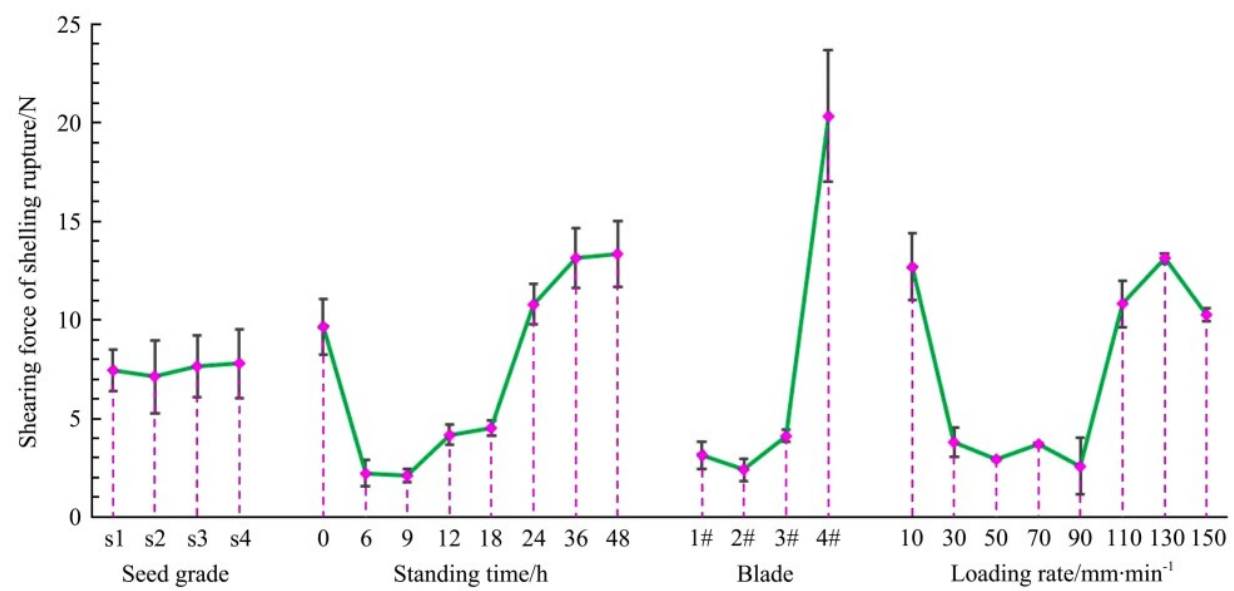

Figure 11 Influence of the factors of the loading mode, seed grade, loading rate, and standing time on the shearing force of shelling

It was found that the shearing force of shelling was minimal $(2.11 \mathrm{~N})$ when the standing time was $9 \mathrm{~h}$ and increased drastically afterward with extended-standing time due to intensified moisture loss of the shell. Fisher's LSD test showed that no significant difference existed in the shearing force between seeds with a standing time of $6 \mathrm{~h}$ and $9 \mathrm{~h}$. In the current mechanical shelling process, the blade firstly cuts a circle along the short axis, and the seed is then pressed and rubbed to separate the kernel from the shell. It was found that when the standing time of the seed was more than $6 \mathrm{~h}$, the separation of the kernel from the shell was much less successful because the shell had softened due to loss of moisture. Therefore, the fresh lotus seed should ideally have a standing time of no more than $6 \mathrm{~h}$.

The shearing force of shelling decreased gradually when the loading rate increased from $10 \mathrm{~mm} / \mathrm{min}$ to $90 \mathrm{~mm} / \mathrm{min}$ and then increased gradually as the loading rate escalated further from $90 \mathrm{~mm} / \mathrm{min}$. Fisher's LSD test showed that no significant difference existed among the four levels of loading rate at 30$90 \mathrm{~mm} / \mathrm{min}$ or among the three levels of loading rate at 110 $150 \mathrm{~mm} / \mathrm{min}$. Therefore, applying a loading rate of $30-90 \mathrm{~mm} / \mathrm{min}$ would help shell the fresh lotus seed.

\section{Conclusions}

There was no obvious point of stress yield during the compression of fresh lotus seed, and the kernel ruptured first before the shell cracked. Fresh lotus seed had greater resistance to compression in the long shaft direction than in the short shaft direction.

Orthogonal tests of compression showed that the influence of individual factors on the compressive force upon kernel rupture ( $p<0.01$ for all factors) ranked in the descending order of loading mode, loading rate, standing time and seed size, and the influence of individual factors on kernel deformation ranked in the descending order of loading mode $(p<0.01)$, standing time $(p<0.01)$, the loading rate $(p<0.01)$ and seed size $(p<0.05)$.

Orthogonal tests of shearing showed that fresh lotus seeds were far more easily shelled by shearing than cracked by compression. The influence of individual factors on shearing rupture force ranked in the descending order of blade angle $(p<0.01)$, standing time $(p<0.01)$, loading rate $(p<0.01)$ and seed size $(p>0.05)$. Seed size had minimal influence on the shearing rupture force.

\section{Acknowledgements}

This work was financially supported by the National Natural
Science Foundation (Grant No. 31772039) and the Special Fund for Key Program of Science and Technology of Fujian (Grant No. 2018NZ003).

\section{[References]}

[1] Luo H L, Liu X X, Huang X Y, Dai X M, Zhang M, Fang F F, et al. Chemical deterioration of lotus seeds during storage. Journal of Food Quality, 2016; 39(5): 496-503.

[2] Zhu M, Liu T, Zhang C, Guo M. Flavonoids of Lotus (Nelumbo nucifera) seed embryos and their antioxidant potential. Journal of Food Science, 2017; 82(3): 1834-1841.

[3] Liu T, Zhu M, Zhang C, Guo M. Quantitative analysis and comparison of flavonoids in lotus Plumules of four representative lotus cultivars. Journal of Spectroscopy, 2017; 2017: 1-9.

[4] Fujian Provincial bureau of statistics. The statistical yearbook of Fujian Province (2018): 10-16 Non-grain crop yield, 2018. (in Chinese)

[5] Luo Y H, Rao X X, Yang S C. New processing technology of lotus seed of Jianning. China Vegetables, 2011; 11: 46-48. (in Chinese)

[6] Chen C Q, Zhu J X, Zheng T, Lin X, Wang J, Fei Y. Research status and existing problems on mechanized processing of fresh lotus seeds. Agricultural Development \& Equipments, 2018; 24(7): 103-104. (in Chinese)

[7] Liang S, Lin Y, Fan Z, Zhang J, He J. Progress and discussion of fresh lotus seed shelling technology. Hubei Agricultural Sciences, 2017; 56(4): 603-607. (in Chinese)

[8] Chen Y, Xiang H, Tan J, Zou X, Huang G, Li B. Effects of extrusion on mechanical damage and mechanical parameters of litchi. Journal of Hunan Agricultural University (Natural Sciences), 2013; 39(6): 688-692. (in Chinese)

[9] Chen Y, Cai W, Zou X, Xiang H, Liu T, Xu F. Mechanical properties test and finite element analysis for litchi. Transactions of the CSAE, 2011; 27(12): 358-363. (in Chinese)

[10] Wang W, Zhang S, Fu H, Lu H, Yang Z. Evaluation of litchi impact damage degree and damage susceptibility. Computers and Electronics in Agriculture, 2020; 173: 105409. doi: 10.1016/j.compag.2020.105409.

[11] Xue J, Gao S, Fan Y, Li L, Ming B, Wang K, et al. Traits of plant morphology, stalk mechanical strength, and biomass accumulation in the selection of lodging-resistant maize cultivars. European Journal of Agronomy, 2020; 117: 126073. doi: 10.1016/j.eja.2020.126073.

[12] Johnson P C, Clementson C L, Mathanker S K, Grift T E, Hansen A C. Cutting energy characteristics of Miscanthus x giganteus stems with varying oblique angle and cutting speed. Biosystems Engineering, 2012; 112(1): 42-48.

[13] Mathanker S K, Grift T E, Hansen A C. Effect of blade oblique angle and cutting speed on cutting energy for energycane stems. Biosystems Engineering, 2015; 133: 64-70.

[14] Pérez-López A, Chávez-Franco S H, Villaseñor-Perea C A Espinosa-Solares T, Hernández-Gómez L H, Lobato-Calleros C. Respiration rate and mechanical properties of peach fruit during storage at three maturity stages. Journal of Food Engineering, 2014; 142(6): 111-117. 
[15] Wang H, Liu C, Xue Y, Li D. Correlation of mechanical properties of peach slices with cell wall polysaccharides and cell morphology during hot air predrying. Journal of Food Processing and Preservation, 2020; 44(2): e14319. doi: 10.1111/jfpp. 14319 .

[16] Ekrami-Rad N, Khazaei J, Khoshtaghaza M H. Selected mechanical properties of pomegranate peel and fruit. International Journal of Food Properties, 2011; 14(3): 570-582.

[17] Delprete C, Giacosa S, Raviolo E, Rolle L, Sesana R. Experimental characterization and numerical modeling of the compressive mechanical behavior of hazelnut kernels. Journal of Food Engineering, 2015; 166: 364-369.

[18] Xie L, Li Z, Li X. Experimental study on mechanical properties of lotus seed. Transactions of the CSAE, 2005; 21(7): 11-14. (in Chinese)

[19] Ye X. Testing and analyzing of physical properties of white lotus seed and red lotus seed. Anhui Agricultural Science Bulletin, 2006; 12(5): 69-70. (in Chinese)

[20] Zhao X, Zong L, Xie L. Testing and analyzing of physical parameters of dry white and red lotus. Food \& Machinery, 2006; 22(2): 53-55. (in Chinese)
[21] Xie L, Zong L. Analysis of finite element method for loaded lotus seed. Transactions of the CSAM, 2006; 37(6): 94-97. (in Chinese)

[22] Zhao J. Study on the structure and properties of plant nut materials and their bionics significance. Master dissertation. Tianjin: Tianjin University, 2007; 71p.

[23] Zhou Y. Research on physical-mechanical properties and cutting processing properties of lotus kernels. Master dissertation. Xiangtan: Xiangtan University, 2014; 65p. (in Chinese)

[24] Ma Q C, Lu A, Chen K, Zhao F, Lei L, Zhang J. Study on self-adaptive centering method of removing core of lotus seed and prototype test. Transactions of the CSAE, 2014; 30(21): 17-24. (in Chinese)

[25] Ma Q, Guo G, Ma J, Lei L, Liu K, Long H, et al. Determination of mechanical characteristic parameters and extrusion crushing characteristics test for lotus seed kernel. Transactions of the CSAE, 2018; 34(6): 263-271. (in Chinese)

[26] Zhu H, He J, Fang W, Ye D, Liang S. Design and test of small fresh lotus seed sheller. Transactions of the CSAE, 2017; 33(7): 28-35. (in Chinese)

[27] Zhang S, Peng Y, Zong L. Shearing mechanical properties of fresh lotus. Guangdong Agricultural Sciences, 2014; 41(18): 155-159. (in Chinese) 